\title{
Explicit and implicit method in nonlinear seismic analysis
}

\author{
Ivan Němec ${ }^{1, *}$, Hynek Štekbauer ${ }^{1}$, Adéla Vaněčková $^{1}$ and Zbyněk Vlk $^{1}$ \\ ${ }^{1}$ Brno University of Technology, Faculty of Civil Engineering, Institute of Structural Mechanics, \\ Veveř́ 331/95, 60200 Brno, Czech Republic
}

\begin{abstract}
The paper deals with suitability of use of the explicit and implicit method in heavily nonlinear seismic analysis. Considering typical time of duration of earthquakes from several seconds to approximately twenty seconds, it would seem that use of the implicit method is definitely more suitable. The shape of accelerograms, however, requires quite short time steps. For the explicit method, on the other hand, very short time steps are required in order to obtain a stable solution. Both methods must be compared with respect to a heavily nonlinear response, typical for seismicity. Conclusions of the paper are made on the basis of detailed numerical study and may be very useful for practice.
\end{abstract}

\section{Numerical methods of direct integration of equation of motion}

In the following text let us introduce the numerical methods used in the study. A system of motion equations of a discrete model of a structure subjected to a dynamic load can be written

$$
\mathbf{M a}(t)+\mathbf{C v}(t)+\mathbf{K u}(t)=\mathbf{F}(t)
$$

Numerical methods of direct integration solve system (1) in a finite number of time instants $t_{0}, t_{1}, \ldots, t_{m}$. The distance between individual time instants $\Delta t_{i}=t_{i}-t_{i-1}$ is called the length of the integration step. The lengths of integration steps $\Delta t_{i}$ influence the accuracy, stability and speed of the solution. Defined initial conditions are an integral part of system (1). The time $t=0$ is considered to be the starting point at which $\mathbf{u}\left(t_{0}\right)=\mathbf{u}_{0}$, $\mathbf{v}\left(t_{0}\right)=\mathbf{v}_{0}$. System (1) can be thus written as

$$
\mathbf{M a} \mathbf{a}_{i}+\mathbf{C} \mathbf{v}_{i}+\mathbf{K u} \mathbf{u}_{i}=\mathbf{F}_{i}
$$

\subsection{Explicit methods}

In explicit methods we make use of the assumption about the distribution of motion characteristics $\mathbf{u}, \mathbf{v}=\dot{\mathbf{u}}, \mathbf{a}=\ddot{\mathbf{u}}$ in interval $\left\langle t_{i}, t_{i+1}\right\rangle$ and the knowledge of these characteristics

\footnotetext{
* Corresponding author: nemec@,fem.cz
} 
at time instant $t_{i}$, and we calculate vectors $\mathbf{u}_{i+1}, \mathbf{v}_{i+1}, \mathbf{a}_{i+1}$ from (2). Neither triangulation nor modification of the stiffness matrix is performed in explicit methods.

\subsubsection{Method of central differences}

The numerical integration of differential equations uses the substitution of the derivative of the independent variable with respect to time. If we replace the derivatives in (2) by

$$
\begin{gathered}
\mathbf{v}_{i}=\frac{1}{2 \Delta t_{i}}\left(\mathbf{u}_{i+1}-\mathbf{u}_{i-1}\right) \\
\mathbf{a}_{i}=\frac{1}{\Delta t_{i}^{2}}\left(\mathbf{u}_{i+1}-2 \mathbf{u}_{i}+\mathbf{u}_{i-1}\right)
\end{gathered}
$$

we get a recurrent formula for $\mathbf{u}_{i}$

$$
\left(\frac{1}{\Delta t_{i}^{2}} \mathbf{M}+\frac{1}{2 \Delta t_{i}} \mathbf{C}\right) \mathbf{u}_{i+1}=\mathbf{F}_{i}-\left(\mathbf{K}-\frac{2}{\Delta t_{i}^{2}} \mathbf{M}\right) \mathbf{u}_{i}-\left(\frac{1}{\Delta t_{i}^{2}} \mathbf{M}-\frac{1}{2 \Delta t_{i}} \mathbf{C}\right) \mathbf{u}_{i-1}
$$

The method has all the advantages of explicit methods as long as $[\mathbf{C}]=[\mathbf{0}]$ or $[\mathbf{C}]=\alpha[\mathbf{M}]$. Its application is most effective for diagonal mass matrix. However, the method is only conditionally stable. The length of the integration step must meet the condition

$$
\Delta t_{i} \leq \frac{T_{n}}{\pi}
$$

where $T_{n}$ is the smallest vibration period.

\subsection{Implicit methods}

Implicit methods are based on system (2) at time instant $t_{i}$. The numerical integration of the system is carried out step by step using the following formula

$$
\mathbf{a}_{i}=f\left(t_{i}, \mathbf{F}_{i}, \mathbf{u}_{i-1}, \mathbf{v}_{i-1}, \mathbf{a}_{i-1}\right)
$$

with the necessity - in order to be able to start with the solution - to evaluate the acceleration at the beginning of the motion at time $t_{0}$ directly from system (2)

$$
\mathbf{M a} \mathbf{a}_{0}+\mathbf{C} \mathbf{v}_{0}+\mathbf{K u} \mathbf{u}_{0}=\mathbf{F}_{0}
$$

\subsubsection{Newmark methods}

The basic formula of the Newmark method that specifies the relations between displacement, velocity and acceleration vectors have the following form

$$
\begin{gathered}
\mathbf{u}_{i}=\mathbf{u}_{i-1}+\Delta t_{i} \mathbf{v}_{i-1}+\left(\frac{1}{2}-\beta\right) \Delta t_{i}^{2} \mathbf{a}_{i-1}+\beta \Delta t_{i}^{2} \mathbf{a}_{i} \\
\mathbf{v}_{i}=\mathbf{v}_{i-1}+(1-\gamma) \Delta t_{i} \mathbf{a}_{i-1}+\gamma \Delta t_{i} \mathbf{a}_{i}
\end{gathered}
$$

where $\beta$ and $\gamma$ are what is termed Newmark's parameters. As formulas

$$
\mathbf{u}_{i}=\mathbf{u}_{i-1}+\Delta \mathbf{u}_{i}, \mathbf{v}_{i}=\mathbf{v}_{i-1}+\Delta \mathbf{v}_{i}, \mathbf{a}_{i}=\mathbf{a}_{i-1}+\Delta \mathbf{a}_{i}
$$


holds we can write the following formula for the vector of acceleration increments and velocity increments

where

$$
\Delta \mathbf{a}_{i}=\Delta \overline{\mathbf{a}}_{i}+\frac{1}{\beta h^{2}} \Delta \mathbf{u}_{i}, \quad \Delta \mathbf{v}_{i}=\Delta \overline{\mathbf{v}}_{i}+\frac{\gamma}{\beta h} \Delta \mathbf{u}_{i}
$$

$$
\Delta \overline{\mathbf{a}}_{i}=\left(-\frac{1}{\beta \Delta t_{i}} \mathbf{v}_{i-1}-\frac{1}{2 \beta} \mathbf{a}_{i-1}\right), \quad \Delta \overline{\mathbf{v}}_{i}=\left(1-\frac{\gamma}{2 \beta}\right) \Delta t_{i} \mathbf{a}_{i-1}-\frac{\gamma}{\beta} \mathbf{v}_{i-1}
$$

The total increments of the displacement (and analogous increments of the velocity, acceleration and force vectors) can be written as

$$
\Delta \mathbf{u}_{i}=\sum_{k=1}^{n_{\text {TIER }}}{ }^{k} \Delta \Delta \mathbf{u}_{i}
$$

Using the substitution according to formulas above into (2) and the obtained relation is modified we get

$$
\left(\frac{1}{\beta \Delta t_{i}^{2}} \mathbf{M}+\frac{\gamma}{\beta \Delta t_{i}} \mathbf{C}+{ }^{k} \mathbf{K}_{\mathrm{T}, i}\right) \cdot{ }^{k} \Delta \mathbf{u}_{i}={ }^{k} \Delta \mathbf{F}_{i}-\mathbf{M} \Delta \overline{\mathbf{a}}_{i}-\mathbf{C} \Delta \overline{\mathbf{v}}_{i}
$$

The bracket on the left hand side of (15) represents what is termed modified stiffness matrix, which can be denoted $\hat{\mathbf{K}}_{i}$. The presented formula can be written in a similar form as

$$
{ }^{k} \Delta \Delta \mathbf{u}_{i}: \begin{cases}\hat{\mathbf{K}}_{i}{ }^{k} \Delta \Delta \mathbf{u}_{i}={ }^{k} \Delta \Delta \mathbf{F}_{i}-\mathbf{M} \Delta \overline{\mathbf{a}}_{i}-\mathbf{C} \Delta \overline{\mathbf{v}}_{i} ; & \text { for } k=1 \\ \hat{\mathbf{K}}_{i}{ }^{k} \Delta \Delta \mathbf{u}_{i}={ }^{k} \Delta \Delta \mathbf{F}_{i} ; & \text { for } k>1\end{cases}
$$

Using this relation (16), it is possible to calculate partial increments of displacement.

\section{Comparison of the used numerical methods}

For this numerical study a wall of six floor building was used. The structure was subjected to the seismic load due to the accelerogram from Umbro-Marchigiana, Italy.

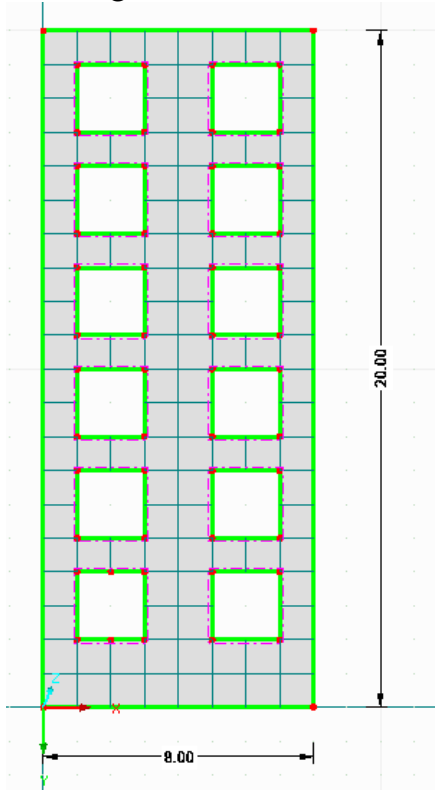

Fig. 1. The analysed structure. 


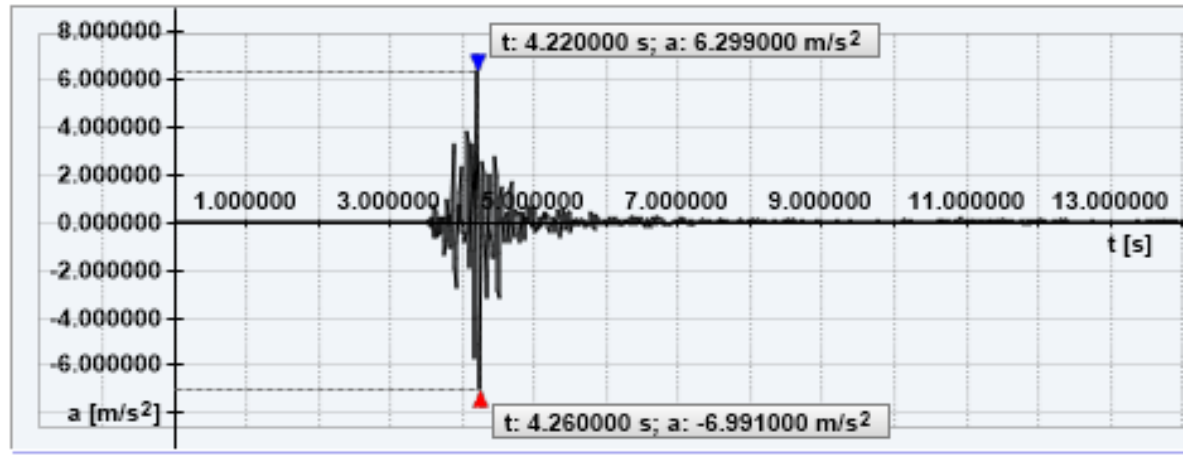

Fig. 2. Accelerograms Umbro-Marchigiana, station Colfiorita-Casermette.

The important aim of the paper is to compare the suitability of use of the explicit method and the Newmark's implicit method in the seismic analysis of structures.

It is commonly known that the explicit method (described e.g. in [1], [2] and [3]) is suitable for actions with very short duration, such as explosions or the impacts of vehicles against obstacles, i.e. actions generally studied via transient dynamic analysis. With the explicit method, the time step necessary from the point of view of computational stability is determined by the shortest time in which sound overcomes the distance between two arbitrary nodes of the structure of the given material. This time step must then be decreased to an even lower duration by a certain safety factor, let's say 0.7 , to be sure that the calculations will be stable. The maximal possible time step then depends on the Young's modulus of the material, the density of the material (the square root of the ratio between the Young's modulus and density is the speed of sound in the material) and on the shortest distance between arbitrary nodes. It can thus be seen that refinement of the mesh will demand a pertinent decrease in the maximum allowed time step. Of course, this will mean an increase in the amount of computational time needed. It is clear from the above conditions that the explicit method requires an extremely short time step. For the analysis of building structures it is usually in the order of $10^{-5} \mathrm{~s}$. Only in the case that the abovementioned conditions are fulfilled is it possible to avoid the necessity of solving a system of linear equations at each time step, and instead solve only one equation of motion for each deformation parameter at each time step. In the case of transient dynamic analysis this is not a substantial disadvantage as, for actions like impacts or explosions, the speed at which changes to the external forces occur also requires very short time steps to be used, so the necessity for such time steps due to computational stability requirements is not an issue.

Implicit methods of solving a set of differential equations (described e.g. in [1], [4], [5] and [6]) are characterized by the fact that a system of linear equations must be solved at each time step. In the case of the dynamic solution of structures, dynamic equilibrium equations are employed. The Newmark implicit method thus ensures the equilibrium conditions of the structure are fulfilled at each time step. This method does not demand such a short time step as is needed in the explicit method, and therefore the implicit method is typically suitable for the solution of dynamical problems of a duration longer than several seconds.

In the case of dynamical tasks like the impact of a vehicle against an obstacle, or the effects of explosions, it is necessary to use a very short time step due to the high speed of load changes. Such a situation also occurs when investigating the response of structures to seismic load. Accelerograms commonly take the form of polygons with the time distance between extremes of acceleration being in the order of thousandths of seconds. The computation of such an accelerogram with sufficiently precise results requires the selection 
of a suitably small time step. This would be an argument for the use of the explicit method. On the other hand, the duration of seismic loading, which varies between seconds and tens of seconds, is a fact supporting the choice of an implicit method, which does not demand so short a time step. Of course, the computational demand for each time step is several times greater compared to that of the explicit method.

Recently, the development of a nonlinear dynamics program module for the RFEM program was completed and this module has now been introduced on the market. Regarding seismic analysis using the direct integration of equations of motion, a substantial improvement compared to competitors has been implemented in the module. Originally, at each time step the acceleration value was obtained by interpolation from the accelerogram and applied in the numerical solution. A study was performed which showed that this approach is not accurate enough because the speed and displacement obtained from numerical time integration from those discrete acceleration values are not accurate enough. A substantial improvement to the algorithm was suggested and implemented. Today, the RFEM program performs precise double time integration right at the beginning of computation in order to obtain the exact time course of displacement from the accelerogram. Additionally, during time step processing, the exact values of displacement in the nodal support are considered instead of the discrete values of acceleration which were used in the previous version of the program. These improvements to the program have been shown to provide a substantial improvement in the accuracy of seismic analysis. This is particularly noticeable in the case of the implementation of Newmark's implicit method in the program. As far as the explicit method is concerned, where the time steps are much shorter than in the implicit method for stability reasons, the improvement of accuracy is not as great as with the implicit method. Nevertheless, some improvement in accuracy has been achieved.

To compare the suitability of the used numerical method for the seismic analysis of a typical building wall, a real accelerogram from Italy was used along with the DruckerPrager material model. The comparison of numerical methods was performed with regard to accuracy and computational performance. A time step of $0.0001 \mathrm{~s}$ was applied in the case of the explicit method due to stability requirements. The same time step was also chosen for the implicit method in order to compare the time requirements of both methods for the same number of time steps, and also to obtain a very precise base solution for accuracy comparisons.

From the following graphs showing the course of the horizontal displacement of the upper right corner of the building it can be seen that there is very good concordance between the results of the explicit and implicit method. This can be regarded as proof that the results of the explicit method are good. The comparison between the implicit solutions from the RFEM and ANSYS programs shows that the results of both programs are practically identical. 


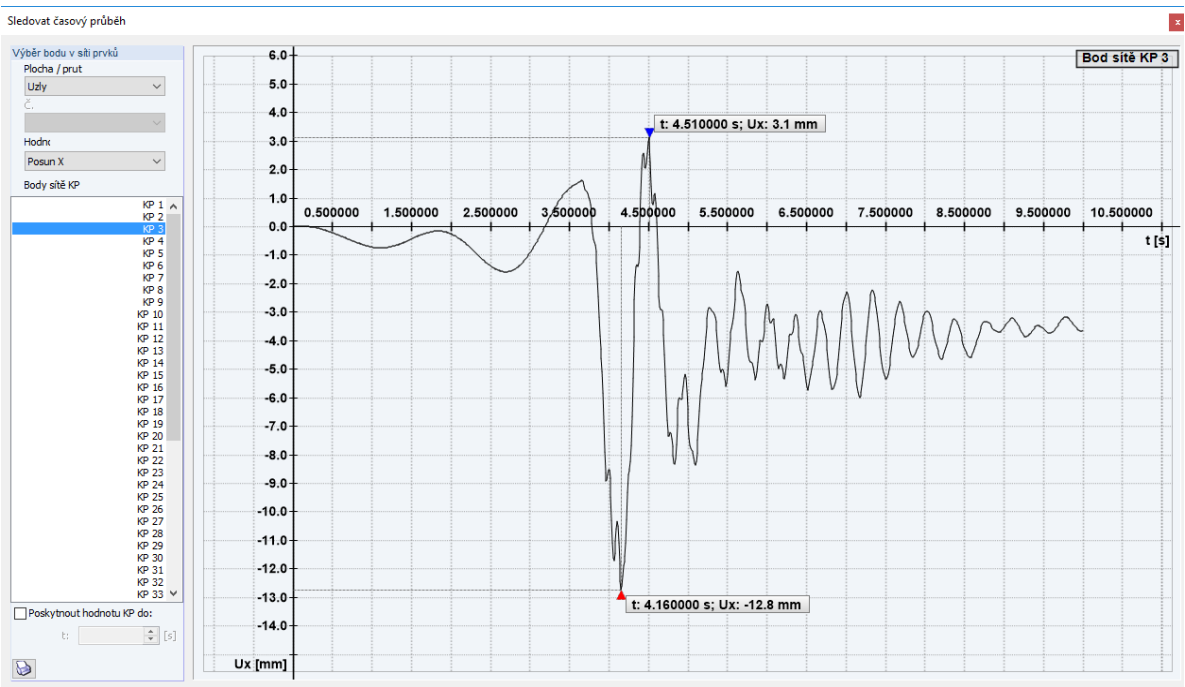

Fig. 3. Explicit method - Graph of the horizontal isplacement of the upper node for the time step $0.0001 \mathrm{~s}$.

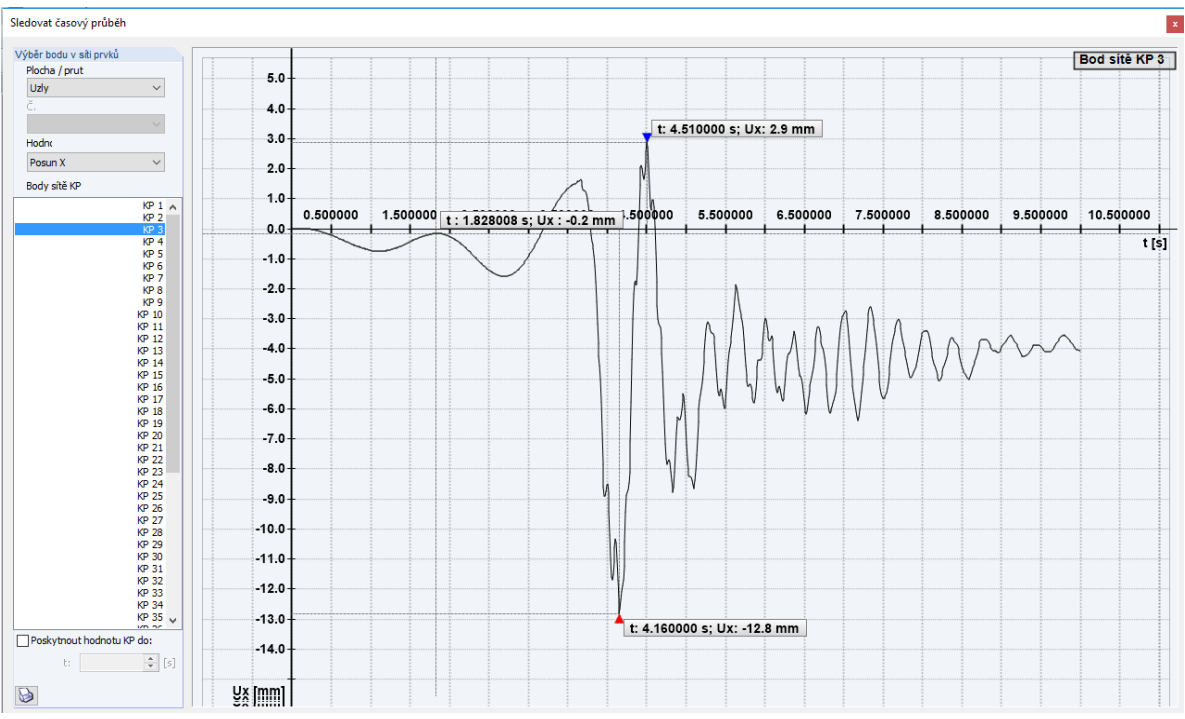

Fig. 4. Implicit method - Graph of the horizontal isplacement of the upper node for the time step $0.0001 \mathrm{~s}$.

\section{Conclusion}

In accordance with expectations, the calculation performed by the implicit method for the same time step as with the explicit method was several times (about 5x) slower than when carried out by the latter method. The time step for the implicit method was then increased fivefold. In this case, the computational time for both methods was practically identical. The results for both solutions were still practically the same (only the magnitudes of the horizontal displacements of the upper right corner were compared). When the time step for the implicit method was increased further, the results provided by the method remained 
accurate enough until the time step was twenty times greater than the time step for the explicit method.

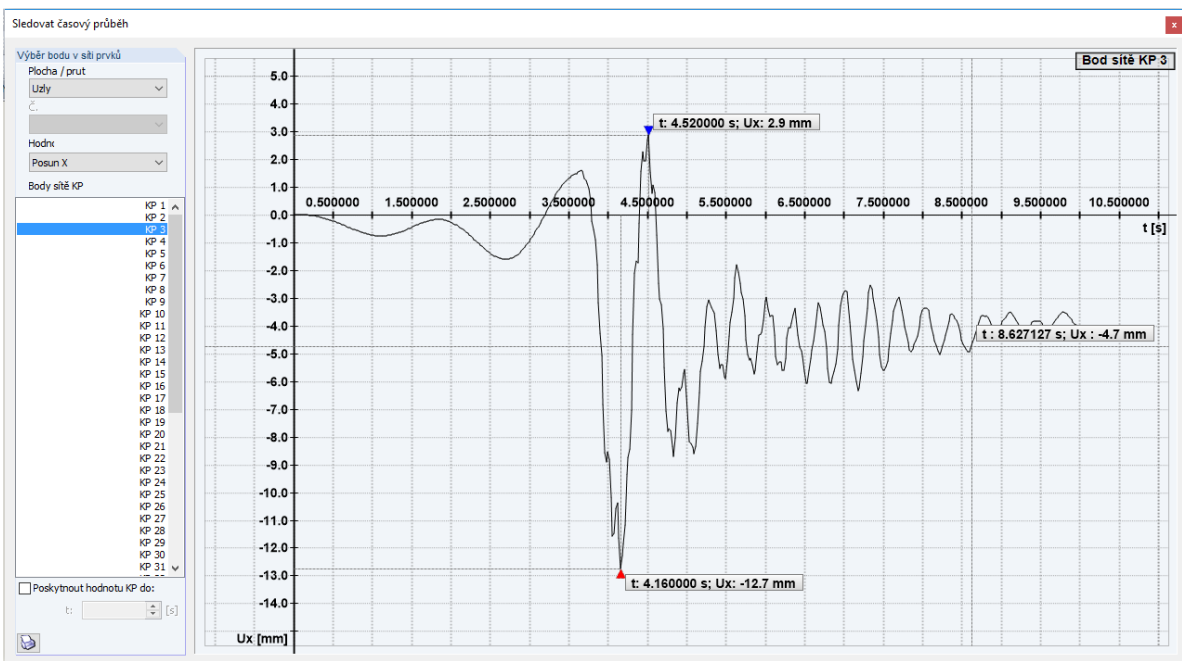

Fig. 5. Implicit method - Graph of the horizontal isplacement of the upper node for the time step $0.002 \mathrm{~s}$.

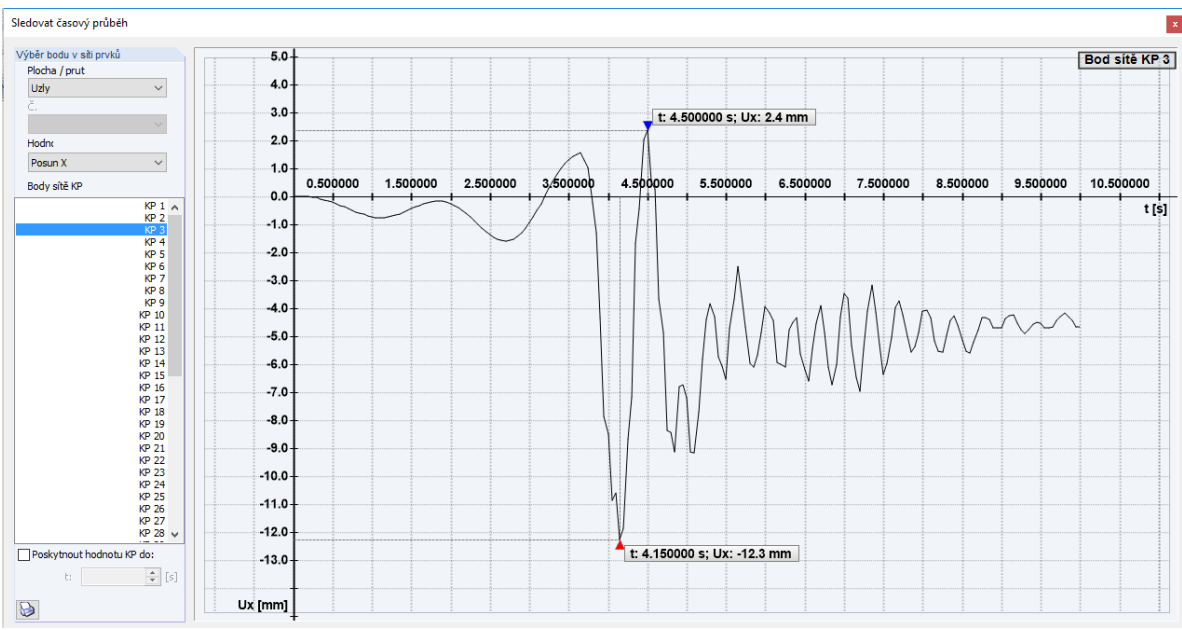

Fig. 6. Implicit method - Graph of the horizontal displacement of the upper node for the time step $0.005 \mathrm{~s}$.

The extreme value of the displacement was only $1 \%$ smaller even in this case, which still can be regarded as acceptable accuracy. Good results were thus obtained from the implicit method for a computational time that was four times lower than that used with the explicit method. Taking into account the fact that it is not possible to estimate the highest acceptable time step for Newmark's implicit method accurately enough without additional effort, it can be accepted that both methods are comparable when used for seismic analysis, though the implicit Newmark method is perhaps the preferred option. The estimation of the highest time step value for the implicit Newmark method is possible with the help of Fourier transformation (in our case the same value was achieved by both of the above tests). However, the process is time-consuming and in practice it will be often easier to use 
a lower time step to be on the safe side, which partially decreases the advantage of the implicit method.

An interesting conclusion of this study is the discovery that the usage of both basic numerical methods for the direct integration of equations of motion, namely the explicit method and Newmark's implicit method, are both competitive in their seismic analysis capabilities. They are also both suitable and comparable as regards practical use.

From the results of numerical analysis it can be seen that there is excellent concordance between the results of the ANSYS and RFEM programs for the implicit method. The concordance demonstrated by the results for the two programs for the explicit method is partially lower, but still acceptable. ANSYS needed some mesh refinement to achieve comparable results.

This research was partially supported by the project FAST-J-17-4720.

\section{References}

1. I. Němec, V. Kolář, I. Ševčík, Z. Vlk, J. Blaauwendraat, J. Buček, B. Teplý, D. Novák, V. Štembera: Finite Element Analysis of Structures, Principles and Praxis (Shaker Verlag, 2010)

2. Shen R. Wu, Lei Gu, Introduction to the Explicit Finite Element Method for Nonlinear Transient Dynamics (John Wiley \& Sons, 2012)

3. J. Rodriguez, G. Rio, J.M. Cadou, J. Troufflard, Numerical study of dynamic relaxation with kinetic damping applied to inflatable fabric structures with extensions for $3 D$ solid element and non-linear behavior, Thin-Walled Structures 49 (11), 1468-1474, Elsevier, (2011)

4. M. Brio, Numerical Time-Dependent Partial Differential Equations for Scientists and Engineers (Academic Press, 2010)

5. K. J. Bathe, Finite element procedures (Prentice Hall, New Jersey, 1996)

6. T. Belytschko, T. J. R. Hughes, Computational methods for transient analysis (Elsevier science publishers B.V, 1983)

7. R. Courant, K. O. Fredrichs, H. Lewy, On the partial difference equations of mathematical physics, IBM Journal of Research and Development 11, (2), 215-234 (1967)

8. J.Alamatian, A new formulation for fictitious mass of the Dynamic Relaxation method with kinetic damping, Computers \& Structures 90-91, 42-54, Elsevier (2012)

9. S. Oller, Nonlinear Dynamics of Structures (Lecture Notes on Numerical Methods in Engineering and Sciences), Springer (2014) 